\title{
Earth-Moon libration point orbit stationkeeping: Theory, modeling, and operations
}

\author{
David C. Folta ${ }^{a, *}$, Thomas A. Pavlak ${ }^{b}$, Amanda F. Haapala ${ }^{b}$, Kathleen \\ C. Howell ${ }^{\text {b }}$, Mark A. Woodard ${ }^{a}$ \\ ${ }^{2}$ NASA Goddard Space Flight Center, Greenbeit, MD, USA \\ ${ }^{\mathrm{S} S c h o o l}$ of Aeronautics and Astronautics, Purdue University, West Lafayette, IN, USA
}

\section{A R T I C L E I N F O}

Article history:

Received 31 July 2012

Received in revised form

17 December 2012

Accepted 29 January 2013

\section{Keywords:}

Stationkeeping

ARTEMIS

Circular restricted three-body problem

Poincaré maps

\begin{abstract}
A B S T R A C T
Collinear Earth-Moon libration points have emerged as locations with immediate applications. These libration point orbits are inherently unstable and must be maintained regularly which constrains operations and maneuver locations. Stationkeeping is challenging due to relatively short time scales for divergence, effects of large orbital eccentricity of the secondary body, and third-body perturbations. Using the Acceleration Reconnection and Turbulence and Electrodynamics of the Moon's Interaction with the Sun (ARTEMIS) mission orbit as a platform, the fundamental behavior of the trajectories is explored using Poincare maps in the circular restricted three-body problem. Operational stationkeeping results obtained using the Optimal Continuation Strategy are presented and compared to orbit stability information generated from mode analysis based in dynamical systems theory.
\end{abstract}

Published by Elsevier Ltd. on behalf of IAA

\section{Introduction}

Earth-Moon collinear libration points have emerged as locations with immediate applications. The selection of Earth-Moon (EM) libration point orbit orientations and amplitudes, as well as the inherent stationkeeping challenges, are correlated and achieving the best result is nontrivial. Thus, this paper offers the Acceleration Reconnection and Turbulence and Electrodynamics of the Moon's Interaction with the Sun (ARTEMIS) mission as a platform to explore: the theory of EM libration point orbit evolution, the required modeling of the libration point orbits, an assessment of Earth-Moon libration point orbit stability,

\footnotetext{
* This paper was presented during the 1st DyCoSS in Porto.

* Corresponding author at: 8800 Greenbelt Rd., Greenbelt, MD 207712400, USA. Tel.: + 1301286 6082; fax: +1 7654940307.

E-mail addresses: david.c.folta@nasa.gov (D.C. Folta),

tpavlak@purdue.edu.(T.A. Pavlak), ahaapala@purdue.edu (A.F. Haapala), howell@purdue.edu (K.C. Howell),

mark.a,woodard@nasa.gov (M.A. Woodard).
}

the use of Poincare maps to define and, potentially, select orbit characteristics, and in-flight stationkeeping strategies. Results using operational data are demonstrated in orbit analysis using Poincaré maps, and in the implementation of stationkeeping strategies as applied to ARTEMIS.

The libration point orbits employed for ARTEMIS are inherently unstable and stationkeeping maneuvers applied approximately once per week are required. The relatively short time scales for divergence, due to the effects of large orbital eccentricity of the secondary and solar gravitational and radiation pressure perturbations, prove challenging for orbital stationkeeping. However, the Optimal Continuation Strategy (OCS), which employs various numerical methods to determine maneuver locations and optimize stationkeeping delta- $V(\Delta V)$, successfully minimizes fuel cost during ARTEMIS mission operations while offering quality navigation tracking and maneuver planning scenarios. To examine libration point orbit control methods, the operationally demonstrated and validated Optimal Continuation Strategy is compared with an implementation of Floquet mode information as calculated from navigation states. The OCS

0094-5765/\$ - see front matter Published by Elsevier Ltd. on behalf of IAA http://dx.dui.org/10.1016/j.actaastro.2013.01.022

Please cite this article as: D.C. Folta, et al., Eat th-Moon libration point orbit stationkeeping: Theory, modeling, and operations, Acta Astronautica (2013), http://dx.do1.org/10 1016/j actaastru.2013 01022 
yields the optimal maneuver locations and $\Delta V$ directions for the given mission constraints and it is demonstrated that these $\Delta V$ directions compare closely with the stable eigenvector directions computed via Floquet analysis.

\section{Background}

\subsection{The ARTEMIS mission}

As the first Earth-Moon libration point orbiting mission, ARTEMIS serves as the basis (investigative platform) for this exploration. More specifically, ARTEMIS is the first mission flown to and continuously maintained in orbit about both collinear Earth-Moon libration points, EM $L_{1}$ and EM $L_{2}$ [1-5]. Originally, five spacecraft comprised the successful Time History of Events and Macroscale Interactions during Substorms (THEMIS) mission. The ARTEMIS mission was characterized by the transfer of the two outermost THEMIS spacecraft from their elliptical Earth orbits and, with lunar gravity assists, re-directed them to the vicinity of EM $L_{2}$ via transfer trajectories that exploited the Sun-Earth multi-body dynamical environment. Two identical ARTEMIS spacecraft, named P1 and P2, entered the space near the Earth-Moon libration point orbits in 2010 on August 25 and October 22, respectively. Once the Earth-Moon libration point orbits were achieved, they were maintained for approximately

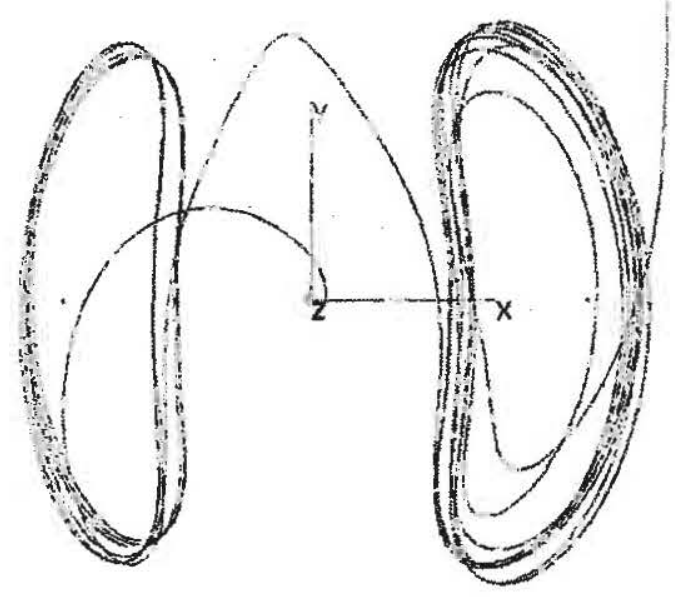

Fig. 1. ARTEMIS P1 libration point orbits, $x-y$ projection.

a

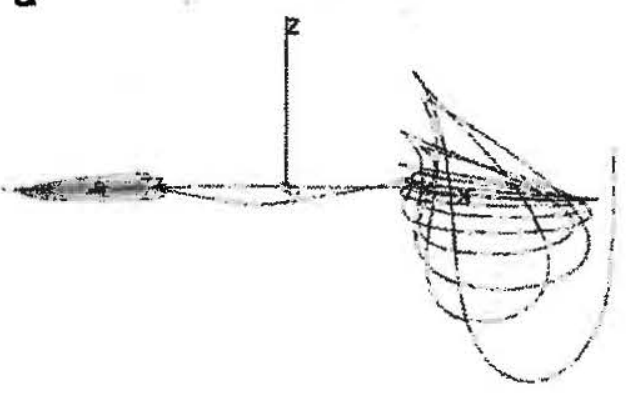

eleven months. The P1 spacecraft was inserted into orbit in the vicinity of EM $L_{2}$ and, during the stationkeeping phase, was transferred from EM $L_{2}$ to EM $L_{1}$. The P2 spacecraft, however, inserted near EM $L_{2}$, but immediately transferred into an EM $L_{1}$ orbit where it was maintained for the remainder of the mission. At the conclusion of the stationkeeping phase, both spacecraft departed their EM $L_{1}$ orbits and inserted into elliptical, low inclination lunar orbits in 2011 on June 27 and July 17, respectively.

Various views of the EM $L_{2}$ and $L_{1}$ libration point orbits associated with the ARTEMIS P1 spacecraft appear in Figs. 1 and 2. Similarly, projections of the ARTEMIS P2 EM $L_{1}$ libration point orbit are depicted in Figs. 3 and 4.

There were no size or orientation requirements on these orbits other than to minimize the libration point orbit insertion and stationkeeping $\Delta V$ costs and, ultimately, to allow for transfer into low inclination lunar orbits. Both ARTEMIS spacecraft had limited (combined) deterministic and statistical stationkeeping $\Delta V$ budgets of $15 \mathrm{~m} / \mathrm{s}$ and $12 \mathrm{~m} / \mathrm{s}$ for $\mathbf{P} 1$ and $\mathrm{P} 2$, respectively. These $\Delta V$ budgets included any libration point orbit stationkeeping requirements as well as the transfers between libration points and into lunar orbit. The amplitudes of the ARTEMIS orbits were governed by the EM $L_{2}$ orbit insertion conditions as dictated by the ballistic Sun-Earth to EarthMoon transfers that were utilized. The resulting P1 and P2 EM $L_{1}$ orbit $y$-amplitudes were approximately $60,000 \mathrm{~km}$

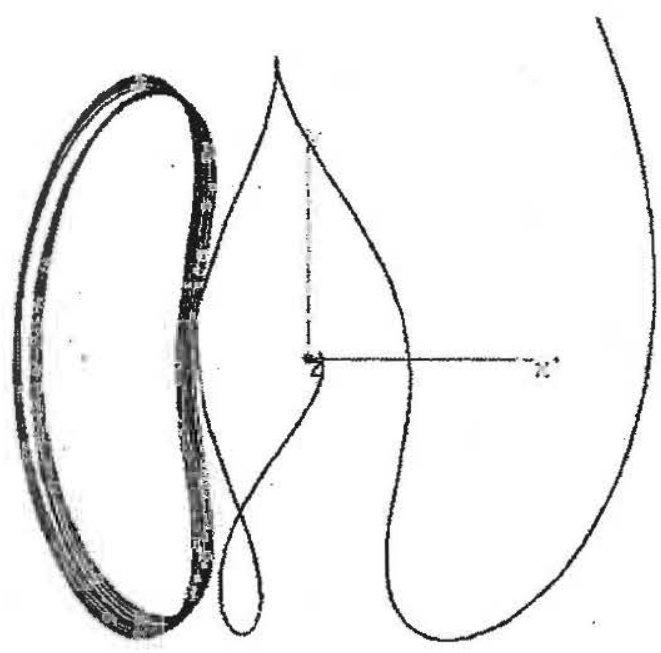

Fig. 3. ARTEMIS P2 libration point orbits, $x-y$ projection. b

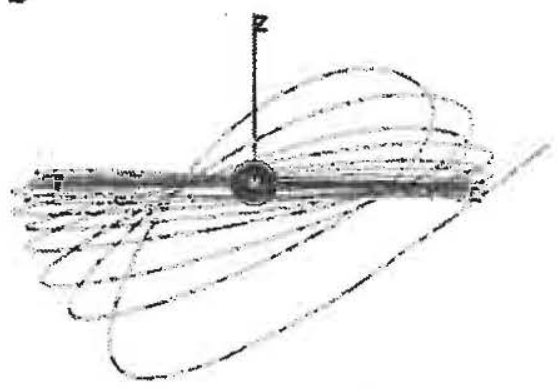

Fig. 2. ARTEMIS P1 libration point orbits, additional projections. (a) $x-z$ projection and (b) $y-z$ projection. 
with the P1 $L_{2}$ orbit $y$-amplitude near $68,000 \mathrm{~km}$. A complete list of the general dimensions for the evolving ARTEMIS libration point orbits appears in Table 1.

\subsection{Environmental modeling}

The ARTEMIS P1 and P2 trajectories, like many collinear libration point orbits, are dynamically unstable and even small perturbations can significantly affect their evolution. During operations, if any inaccuracies in the dynamical model are present, a spacecraft can depart from the desired EM $L_{1}$ or $L_{2}$ orbit along an unstable manifcld, either towards the Moon or along an escape trajectory towards the Earth or, even, away from the Earth-Moon system. The perturbations required to affect these changes are so small that even mis-modeled accelerations due to solar radiation pressure or additional gravitating bodies can result in departure from the nominal libration point orbit. Thus, for successful mission operations, the system must be modeled as a true fourbody problem, including the Sun's gravity as an important third-body perturbation; accelerations due to lunar eccentricity and solar radiation pressure are incorporated as well. The relative effect of these force terms on the evolution of the ARTEMIS P2 trajectory are explored by Pavlak and Howell [6]. Operationally, the ARTEMIS mission trajectory design and analysis utilized a full DE421 Moon-Earth-Sun ephemeris model that also incorporated solar radiation pressure acceleration based upon the spacecraft mass and cross-sectional area - in this case, a simplified cannon ball model was used - and a gravity potential model for the Earth with degree and order eight. The high-fidelity orbit propagation was conducted using both Coddard Space Flight Center's General Mission Analysis Tool (GMAT) and AGI's STK/Astrogator software package. Successful libration point orbit stationkeeping operations rely on high-fidelity modeling to accurately predict trajectory evolution and, ultimately, to design $\Delta V$ maneuvers that ensure the mission objectives are satisfied.

Table 1

ARTEMIS libration point orbits parameters.

\begin{tabular}{llll}
\hline Orbit parameter & P1 at $L_{1}$ & P1 at $L_{2}$ & P2 at $L_{1}$ \\
\hline Maximum x-amplitude $(\mathrm{km})$ & 23,656 & 32,686 & 30,742 \\
Maximum y-amplitude $(\mathrm{km})$ & 58,816 & 63,520 & 67,710 \\
Maximum z-amplitude $(\mathrm{km})$ & 2387 & 35198 & 4680 \\
Orbital period (days) & 13.51 & 15,47 & 14.19 \\
\hline
\end{tabular}

\section{Theoretical background and libration point orbit analysis}

While high-fidelity modeling is important for final mission design and operations, the circular restricted threebody (CR3B) problem is a simplified multi-body model that can provide much insight for pre-flight trajectory planning and offers a very useful perspective during post-mission analysis for ARTEMIS. The libration point orbits for the ARTEMIS mission were selected to minimize libration point orbit insertion and stationkeeping costs and to enable low-cost transfers to low inclination lunar orbits; there were no specific size or orientation requirements on these orbits. Analysis of the libration point orbits from the perspective of the CR3B problem via Poincaré maps offers further insight into the orbital evolution of the ARTEMIS spacecraft trajectories and dynamical evidence that demonstrates why the selected trajectories were ultimately successful in satisfying the mission constraints.

\subsection{The circular restricted three-body problem}

In the CR3B model [7], the motion of a spacecraft, assumed massless, is governed by two gravitationally massive primary bodies, the Earth and the Moon, each represented as a point mass. The orbits of the primary bodies are assumed circular relative to the system barycenter. A barycentered rotating frame is defined such that the rotating $x$-axis is directed from the Earth to the Moon, the $z$-axis is parallel to the direction of the angular velocity of the primary system, and the $y$-axis completes the right-handed triad. The spacecraft state relative to the Earth-Moon barycenter is defined in terms of rotating coordinates as $\boldsymbol{x}=[x, y, z, \dot{x}, \dot{y}, \dot{z}]$. Note that bold symbols denote vector quantities. The mass parameter, $\mu$, is defined

$\mu=\frac{m_{1}}{m_{1}+m_{2}}$

where $m_{1}$ and $m_{2}$ correspond to the mass of the Earth and Moon, respectively. The first-order, nondimensional, vector equation of motion is

$\dot{\boldsymbol{x}}=\boldsymbol{f}(\boldsymbol{x})$

where the vector field, $\boldsymbol{f}(\boldsymbol{x})$, is defined

$\boldsymbol{f}(\boldsymbol{x})=\left[\dot{x}, \dot{y}, \dot{z}, 2 \dot{y}+U_{x},-2 \dot{x}+U_{y}, U_{z}\right]$

The pseudo-potential, $U$, is defined $U(x, y, z)=\frac{1-\mu}{d_{1}}+\frac{\mu}{d_{2}}+$ $\frac{1}{2}\left(x^{2}+y^{2}\right)$ with the nondimensional Earth-spacecraft and Moon-spacecraft distances written as $d_{1}$ and $d_{2}$, respectively. The quantities $U_{x}, U_{y}, U_{z}$ represent partial a

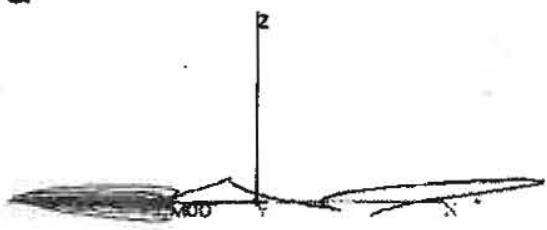

b

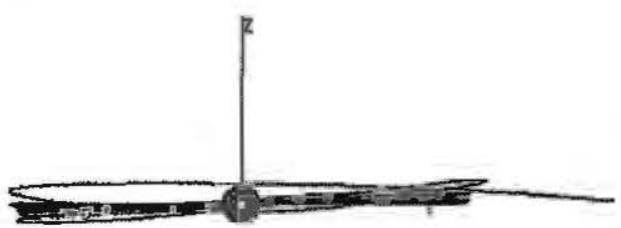

Fig. 4. ARTEMIS P2 libration point orbits, additional projections. (a) $x-z$ projection and (b) $y-z$ projection.

Please cite this article as: D.C. Folta, et al., Earth-Moon libration point orbit stationkeeping: Theory, modeling, and operations, Acta Astronautica (2013), http://dxuloi org/10.1016/j.actaastro.2013.01022 
derivatives of $U$ with respect to rotating position coordinates. The single, scalar integral of the motion, known as the Jacobi constant, $C$, is represented as $C=2 U-v^{2}$, where $v=\left(\dot{x}^{2}+\dot{y}^{2}+\dot{z}^{2}\right)^{1 / 2}$.

Five equilibrium points exist, including three collinear libration points, $L_{1}, L_{2}$, and $L_{3}$, that lie along the $x$-axis, and two equilateral points $L_{4}$ and $L_{5}$. Linear analysis of the collirear points $[7-10]$ reveals that they possess a topological structure of the type saddle $\times$ center $x$ center. Thus, asymptotic flow to and away from the libration points is possible via the stable and unstable manifolds, respectively; periodic and quasi-periodic orbits exist within the center subspace.

\subsection{Libration point orbits and stability}

A linear analysis of the flow in the vicinity of the libration points provides insight into the evolution of libration point orbits in the nonlinear CR3B problem. Linearization of the equations of motion about the collinear libration points allows for states to be selected such that they exist within the center subspace, yielding the following variational equations describing orbits relative to the libration point:

$\delta x(t)=A_{x} \cos (\gamma t+\phi)$.

$\delta y(t)=A_{y} \sin (\gamma t+\phi)$,

$\delta z(t)=A_{z} \sin (\omega t+\psi)$

where $i \gamma, i \omega$ represent the complex eigenvalues associated with the local planar and out-of-plane center manifolds, respectively; angles $\phi, \psi$ represent phase angles and amplitudes $A_{x}, A_{y}$ are related by a proportionality constant. Because $\gamma \neq \omega$, these equations describe quasi-periodic motion in the vicinity of the collinear points whenever $A_{x} \neq 0$ and $A_{z} \neq 0$. Selecting $A_{z}=0$ yields the planar, periodic Lyapunov orbits while choosing $A_{x}=A_{y}=0$ produces the periodic vertical orbits. An example of a quasi-periodic orbit in the linear model appears in black in Fig. 5(a); note that the orbit is linearized relative to the $L_{1}$ point, but is plotted in a Moon-centered view. The $z$-amplitude evolution appears in Fig. 5(b), and illustrates the constant amplitude, $A_{z}$.

By adjusting the phase angles $\phi, \psi$, it is possible to enter the libration point orbit in different locations [11]. As an example, the red segments in Fig. 5 represent shifts in angles $\phi$ and $\psi$ that allow for entry into the orbit at a desired point within the $z$-amplitude evolution cycle.

The variational equations exist within the framework of a linear analysis, however, periodic and quasi-periodic orbits also exist in the full nonlinear model, described by Eqs. (2) and (3) [12]. Several methods exist to compute periodic and quasi-periodic libration point orbits with the inclusion of nonlinear effects [13-17]. A quasi-periodic orbit in the fully nonlinear CR3B problem is depicted in Fig. 6(a); the corresponding $z$-amplitude evolution appears in Fig. 6(b).

Clearly, the $z$-amplitude is no longer constant, but cycles between high and low $z$-amplitude modes. By shifting the entry phasing in the nonlinear model, it is possible to enter the quasi-periodic orbit at a different location and alter the $z$-amplitude mode. The red highlighted region of Fig. 6 illustrates how the entry location in the orbit can be shifted to enter during the nearly planar mode. The entry clearly influences the $z$-amplitude evolution later along the trajectory and/or the maneuver history required to maintain the orbit.

To determine the orbit stability properties of a periodic libration point orbit, the eigenstructure, i.e., eigenvectors and eigenvalues, is analyzed. The monodromy matrix, $M$, is generated by integrating the state transition matrix (STM), $\Phi\left(t_{2}, t_{1}\right)$, along with the trajectory state vector for one orbital period, $T$, i.e., $M=\Phi(t+T, t)$. By computing the 6 eigenvalues, $\lambda_{i}$, of the monodromy matrix, $M$, one can determine the stable eigenvalue(s) $\left(\left|\lambda_{i}\right|<1\right)$, unstable eigenvalue(s) $\left(\left|\lambda_{i}\right|>1\right)$, and eigenvalues reflecting behavior within the center subspace $\left(\left|\lambda_{i}\right|=1\right)$ associated with a particular orbit. An orbit for which all $\left|\lambda_{i}\right|=1$ is considered marginally stable; those orbits for which at least one pair of stable and unstable eigenvalues exist are termed unstable. a

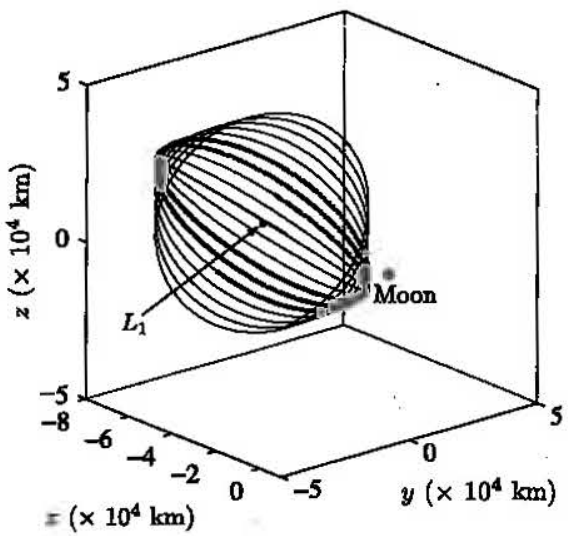

b

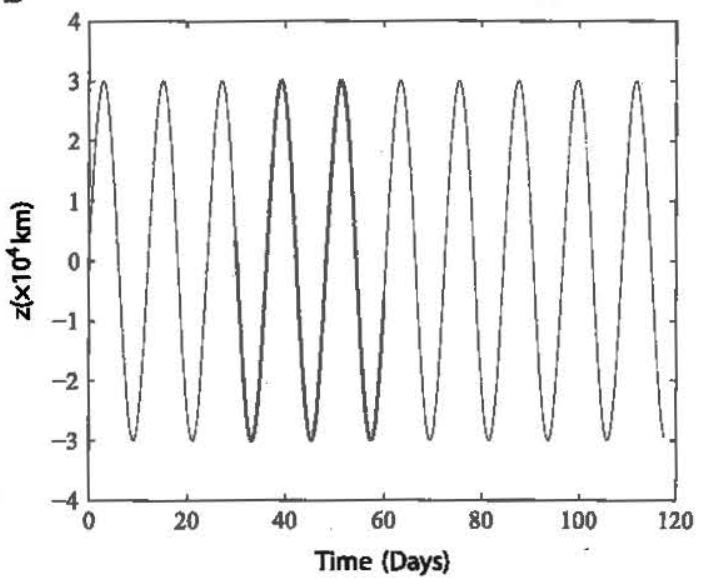

Fig. 5. Quasi-periodic orbit. and corresponding z-amplitude evolution in the linear model. (a) Quasi-periodic orbit in linear model, Moon-centered view and (b) z-amplitude evolution over time. 
a

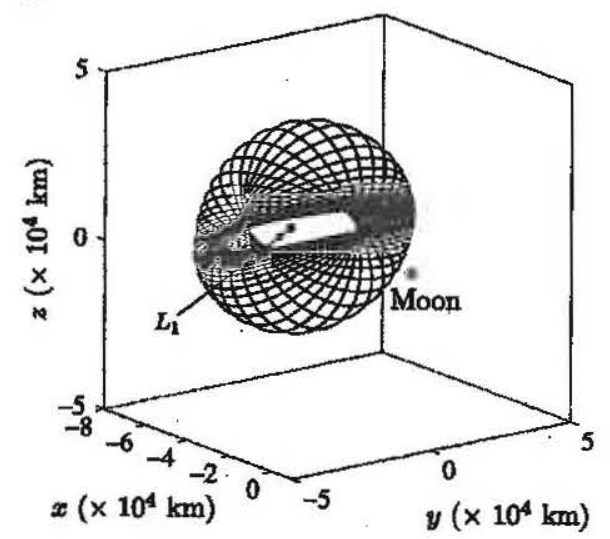

b

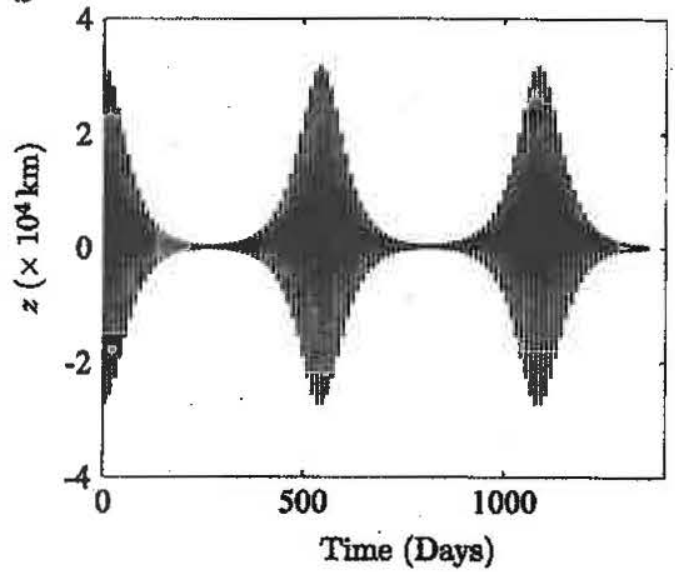

Fig. 6. Quasi-periodic orbit in the CR3B model cycles through high and low z-amplitude modes. (a) Quasi-periodic orbit in CR3B model, Moon-centered view and (b) $z$-amplitude evolution over time.

a

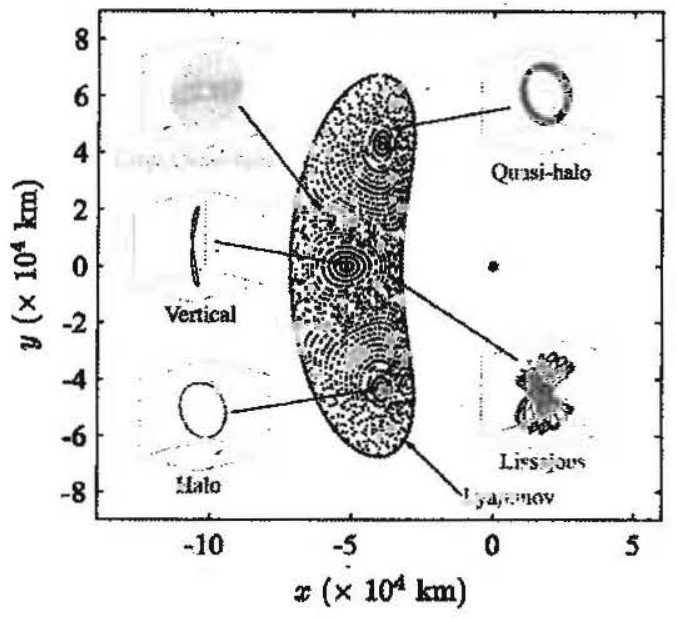

b
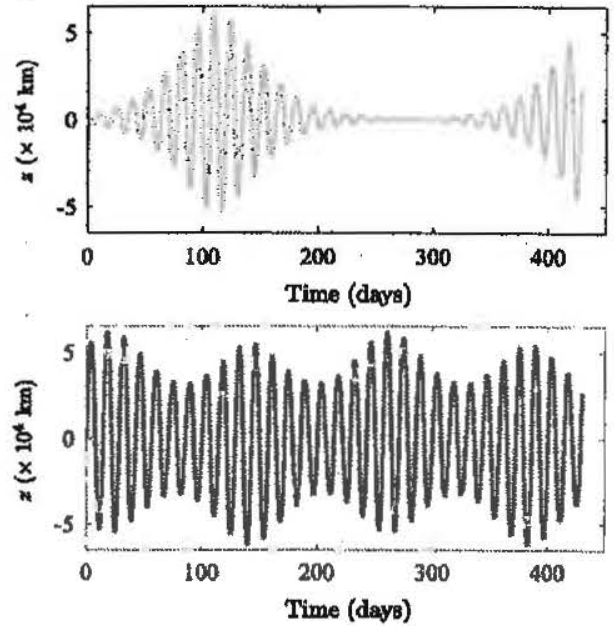

Fig. 7. Poincaré map depicting periodic and quasi-periodic libration point orbit structures in the vicinity of $L_{1}$ in the Earth-Moon system for $C=3.080$. (a) Poincaré map corresponding to crossings of the $x-y$ plane, with sample orbits featured and (b) z-amplitude evolution of selected orbits.

\subsection{Poincaré maps}

For a more complete picture of the available libration point orbits at a particular energy level in the CR3B problem, and for potential design options, it is useful to employ Poincaré maps. Through the use of a Poincaré map, an n-dimensional continuous-time system is reduced to a discrete-time system of $(n-1)$-dimensions. By additionally constraining the Jacobi constant, $C$, the problem is reduced to $(n-2)$-dimensions and, thus, the map for the CR3B problem is represented in 4-D. To generate a planar Poincaré map, a surface-of-section, $\Sigma$, is defined such that $\Sigma$ is transverse to the flow. A commonly used $\Sigma$ is one that represents a surface-of-section corresponding to crossings of the $x-y$ plane. To compute the map, trajectories are integrated using Eqs. (2) and (3), and crossings of $\Sigma$ are recorded and displayed.

Consider the map in Fig. 7 as projected into the $x-y$ plane, produced to resemble the maps demonstrated by
Gómez et ai. [18], as well as Kolemen et al. [16] For the selected value of $C$, several periodic orbits exist, including a planar Lyapunov orbit (green), a vertical orbit (dark blue), and the northern and southern halo orbits. In the $x-y$ projection, the halo orbits share the same crossings of the map; the northern halo is featured in magenta in Fig. 7(a). Utilizing multiple shooting and 30-50 "stacked revolutions" of the periodic orbits, quasi-periodic regions of the Poincaré map are populated using a numerical continuation scheme that begins at the map crossing associated with a periodic orbit and steps outward to yield successively larger quasi-periodic trajectories. Surrounding the periodic vertical orbits are quasi-periodic orbits, often denoted Lissajous orbits, that exist within the center subspace associated with the vertical orbit. A sample Lissajous trajectory is featured in cyan. Similarly, the quasi-halo orbits lie in the center manifold of the central halo orbit. Examples of small and large northern quasi-halo orbits appear in red and orange, respectively. 
The z-amplitude evolutions corresponding to the large quasi-haio and Lissajous orbits featured in Fig. 7(a) appear in Fig. 7(b). The periodic halo and vertical orbits possess constant amplitudes $A_{z}$, whereas the quasi-periodic orbits exhibit oscillating values of $A_{\mathrm{z}}$. The crossings of the Lissajous orbits are contained within the central region of the map; therefore, these orbits do not possess the nearly planar modes demonstrated in Fig. 6 that are desired for ARTEMIStype libration point trajectories. The quasi-halo orbit crossings occur in the upper and lower regions of the map, thus, both high and low $z$-amplitude modes are facilitated by selection of a quasi-halo orbit for this value of $C$. These distinct regions of quasi-periodic behavior are explored in detail by Barden and Howell [19].

\subsection{ARTEMIS Poincaré maps}

To gain insight into the types of orbits selected for ARTEMIS, Poincare maps are generated to display the specific crbit structures associated with the libration point orbit €nergy levels associated with the P1 and P2 spacecraft while in libration point orbits. The ARTEMIS trajectories were designed with higher-fidelity ephemeris modeling, and the true paths possess discontinuities in the form of small $\Delta V^{\prime}$ maneuvers. Thus, to analyze the libration point orbits using maps, it is desirable to compute orbits in the CR3B model that are qualitatively similar to those in the ARTEMIS mission, as depicted in Figs. 1-4. Analogues to the ARTENIS P1 and P2 trajectories are computed in the CR3B problem as explained by Pavlak and Howell [20]. To summarize the approach, an initial guess is developed by first "stacking" planar Lyapunov orbits for a desired number of revolutions about $L_{2}$ and/or $L_{1}$. Stable and unstable manifcld segments are incorporated to transfer between $L_{2}$ anc $L_{1}$ libration point orbits and, ultimately, from the vicinity of $L_{1}$ to the Moon. A multiple shooting algorithm is then utilized to compute continuous, end-to-end trajectories in the CR3B problem with starting points that are consistent with the ARTMEMIS $L_{2}$ orbit insertion states obtained from operational navigation data. For each of the converged trajectories, the Jacobi value, $C$, is evaluated and maps are generated that correspond to the appropriate libration point and the specified energy level. Because the ARTEMIS orbits and the converged CR3B libration point orbits appear qualitatively the same, the results from the discussion of the CR3B orbits can be applied to the actual ARTEMIS orbits as well.

Maps for the P1 $L_{2}, P 1 L_{1}$, and P2 $L_{1}$ libration point orbits appear in Figs. 8-10, respectively. Clearly, each of the three ARTEMIS libration point orbits possesses $x-y$ plane crossings (in red) that lie in the outermost quasi-halo region of the map, indicating that each of the ARTEMIS orbits is a southern large quasi-halo orbit. To demonstrate the long-term evolution of the ARTEMIS trajectories, quasi-halo orbits with map crossings that lie close to each of the ARTEMIS libration point orbit crossings are selected and appear in Figs. 8(b), $9(\mathrm{~b})$, and $10(\mathrm{~b})$, in addition to the crossings associated with the converged ARTEMIS CR3B trajectories plotted in red. The selected quasi-halo orbit crossings are highlighted in color on the maps, and clearly lie close to the ARTEMIS spacecraft crossings. For both the ARTEMIS P1 and P2 trajectories, recall that the libration point orbit insertion conditions are dictated by the ballistic Sun-Earth to Earth-Moon transfers that were utilized and that the orbits were selected, in part. to minimize the $\Delta V$ required to transfer between $L_{2}$ and $L_{1}$ and, eventually, into low inclination lunar orbits. Analysis of the large quasi-halo orbits demonstrates that these orbits supply both high and low z-amplitude phases. Note that the Lissajous family of quasi-periodic orbits does not provide solutions with the near-planar phases that are required based on the ARTEMIS mission constraints. Thus, the ARTEMIS P2 $L_{2}$ trajectory is consistent with the large quasi-halo trajectories because they facilitate both a highly out-of-plane insertion condition and a nearly planar departure to the EM $L_{1}$ side of the Moon. After departing the $L_{2}$ quasi-halo within the nearly planar phase, P1 enters a large $L_{1}$ quasi-halo, but employs only the low $A_{z}$ phase of the orbit to allow for the eventual transfer into a low inclination
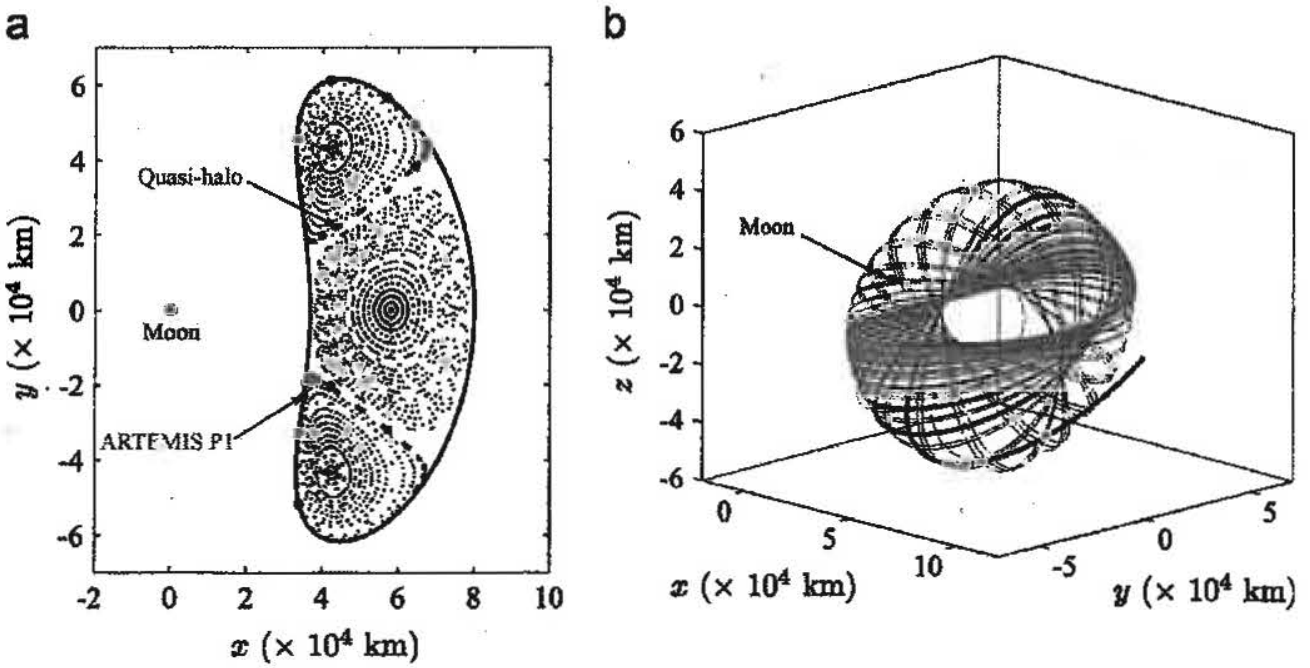

Fig. 8. Poincaré map associated with the ARTEMIS P1 $L_{2}$ orbit ( $C=3.105$ ). (a) Map corresponding to P1 $L_{2}$ orbit and (b) P1 $L_{2}$ orbit (red) with southern quasi-halo of similar size (purple). (For interpretation of the references to color in this figure caption, the reader is referred to the web version of this article.)

Please cite this article as: DC. Folta, et al., Earth-Moon libration point orbit stationkeeping Theory, morleling, and operations, Acta Astronautica (2013), http://dx doi.org/10 1016/j.actaastro 2013.01.022 
a

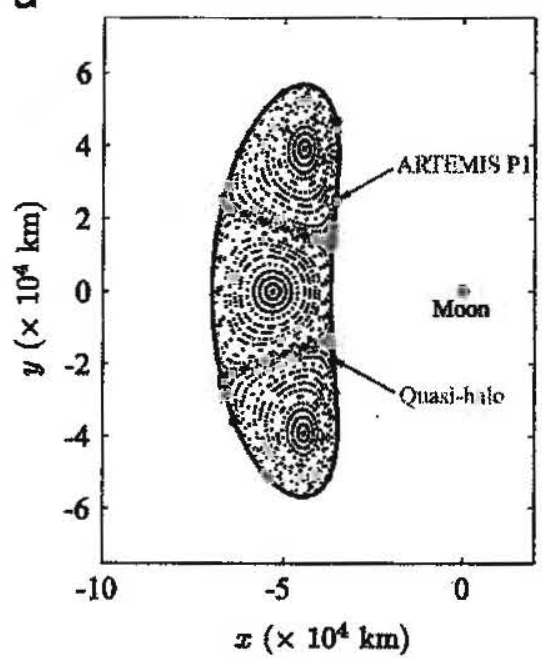

b

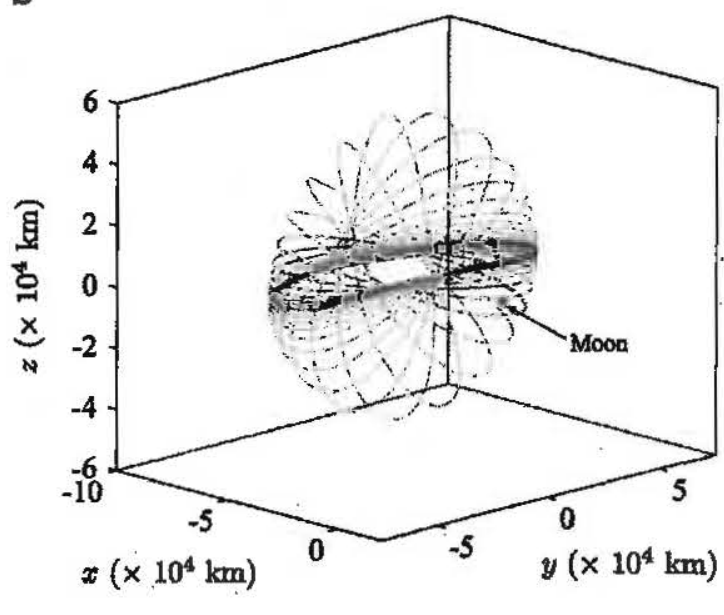

Fig. 9. Poincaré map associated with the ARTEMIS P1 $L_{1}$ orbit $\left(C=3.105\right.$ ). (a) Map corresponding to P1 $L_{1}$ orbit and (b) P1 $L_{1}$ orbit (red) with southern quasihalo of similar size (green). (For interpretation of the references to color in this figure caption, the reader is referred to the web version of this article.)

a

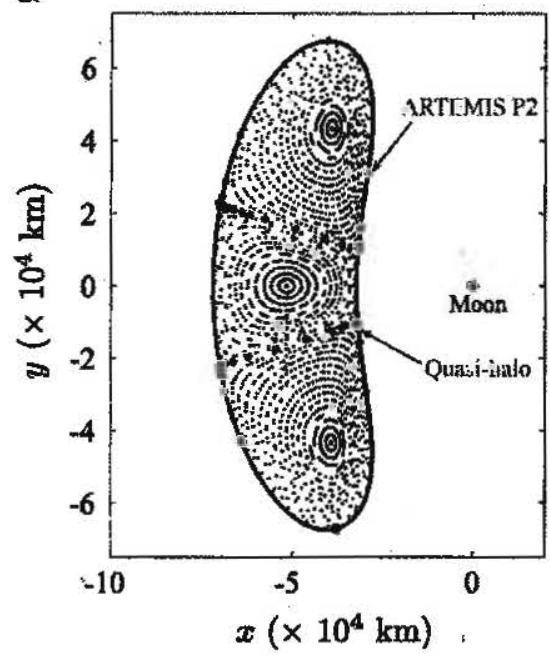

b

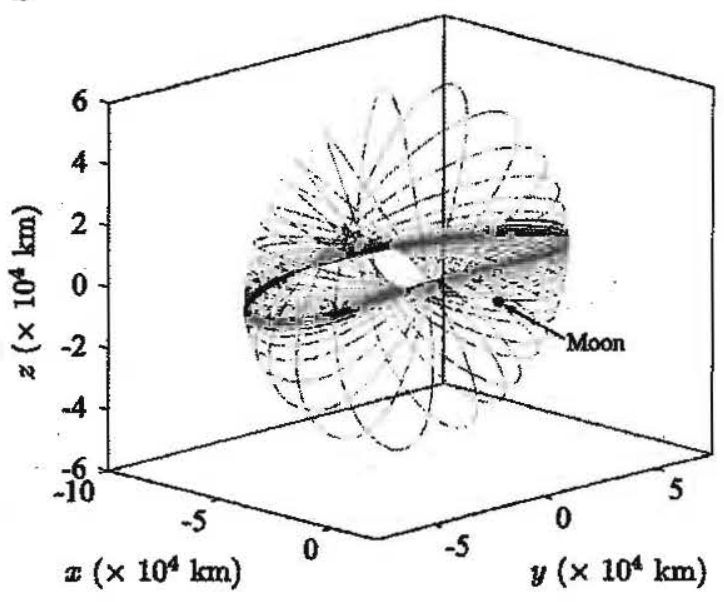

Fig. 10. Poincaré map associated with the ARTEMIS P2 $L_{1}$ orbit ( $C=3.080$ ). (a) Map corresponding to $P 2 L_{1}$ orbit and (b) P2 $L_{1}$ orbit (red) with southem quasihalo of similar size (blue). (For interpretation of the references to color in this figure caption, the reader is referred to the web version of this article.)

lunar orbit. The arrival conditions provided by the ballistic Sun-Earth to Earth-Moon transfer phase for the ARTEMIS P2 spacecraft possess a much smaller out-of-plane component when compared with P1, however, the requirement to transfer to a low inclination lunar orbit remains. Thus, ARTEMIS P2 also exploits the low $A_{z}$ phase of the large $L_{1}$ quasi-halo to reduce the $\Delta V$ costs associated with insertion into a low inclination lunar orbit.

\section{Libration point orbit stationkeeping strategies}

A variety of stationkeeping strategies have previously been investigated for applications in the Sun-Earth system and near the Earth-Moon libration points. To be operationally useful for the ARTEMIS mission, a stationkeeping strategy must satisfy several conditions: utilize highfidelity ephemeris models, yield optimal solutions, and be applicable to Earth-Moon $L_{1}$ or $L_{2}$ libration point orbits and any transfer between them. There were no specific constraints on the size or evolution of the ARTEMIS trajectories as long as the mission constraints were satisfied so stationkeeping strategies that require strict adherence to a reference trajectory were not well-suited to this application. Ultimately, the stationkeeping approach selected for the ARTEMIS mission was able to incorporate high-fidelity modeling of the Earth-Moon dynamical environment and accommodate spacecraft constraints while meeting mission objectives.

\subsection{Previous stationkeeping strategies}

Numerous references in the literature offer discussions of stability and control for vehicles at both collinear and triangular libration point locations. Hoffman [21] and 
Farquhar [22] both generate analysis and discussions for stability and control of spacecraft in Earth-Moon collinear $L_{1}$ and $L_{2}$ locations, respectively, within the context of classical control theory and/or linear approximations. Renault and Scheeres offer a statistical analysis approach [23]. Howell and Keeter [24] address the use of Floquet theory to select stationkeeping maneuvers to eliminate the unstable modes associated with a reference orbit: Gómez et al. [25] independently develop and apply the Floquet mode approach specifically to translunar libration point orbits. Marchand and Howell [26] discuss stability including the eigenstructures near the Sun-Earth locations. Folta and Vaughn [27] present an analysis of staticnkeeping options and transfers between the EarthMoon locations, and the use of numerical models that include discrete linear quadratic regulators and differential correctors. Pavlak and Howell [20] demonstrate a Iong-ierm orbit maintenance technique incorporating multiple shooting. Lastly, Folta et al. $[1,2,4]$, Woodard et al. [3], and Sibeck et al. [5] provide both a review of various Earth-Moon libration point orbit stationkeeping methods, as well as detailed operational stationkeeping and transfer results for the ARTEMIS mission.

\subsection{Optimal Continuation Strategy (OCS)}

Based on a comparison of existing libration point orbit stationkeeping methods by Folta et al. $[1,2]$ and the imposed operational constraints, it was determined that the Optimal Continuation Strategy (OCS) offered a flexible method for maintaining the ARTEMIS Earth-Moon libration point orbits. Fundamentally, the OCS is designed to maintain the spacecraft in the vicinity of a libration point orbit for 1-2 revolutions downstream. The OCS achieves this cbjective by computing maneuvers such that the spacecraft satisfies a set of user-defined constraints at successive crossings of the $x-z$ plane. Often, these constraints are expressed in terms of either $x$-position or $x$-velccity. At its foundation, the OCS is formulated as a two-point boundary value problem and can be solved using any appropriate differential corrections scheme. Nonlinear optimization such as sequential quadratic programming (SQP) can also be incorporated to yield locally optimal stationkeeping maneuvers. Initially, the algorithm computes a maneuver to achieve constraints one $x-z$ plane crossing downstream. Once a converged solution is obtained, the targeting procedure is repeated to satisfy constraints at successive future $x-z$ plane crossings - typically 3-4 crossings maximum. The OCS is implemented in this manner at all subsequent stationkeeipng maneuver locations to maintain a libration point orbit for an arbitrary number of revolutions without requiring a reference solution.

\section{Stationkeeping operations for the ARTEMIS mission}

\subsection{ARTEMIS OCS implementation}

The flexible nature of the Optimal Continuation Strategy and the fact that it does not require a reference solution, made it well-suited to ARTEMIS stationkeeping operations. In this implementation of the OCS, constraints on $x$-velocity were incorporated at $x-z$ plane crossings. However, the specific $x$-velocity targets employed in this application differed slightly between the EM $L_{2}$ and $L_{1}$ orbits and were determined empirically during premission analysis [2]. The velocity targets to maintain the P1 spacecraft in orbit about EM $L_{2}$ consisted of two different $x$-velocity targets, depending on the spacecraft location in the orbit, i.e., the side closer or farther from the Moon. For $x-z$ plane crossings on the far side of the $L_{2}$ orbit (farther from the Moon), the OCS targeted an $x$-velocity $-20 \mathrm{~m} / \mathrm{s}$ with a tolerance of $1 \mathrm{~cm} / \mathrm{s}$. Crossing targets on the near side of the $L_{2}$ trajectory (nearer to the Moon), however, required an $x$-velocity $+10 \mathrm{~m} / \mathrm{s}$ with a tolerance of $1 \mathrm{~cm} / \mathrm{s}$. For both the P1 and P2 orbits in the vicinity of EM $L_{1}$, the $x$-velocity, $\dot{x}$, at each $x-z$ plane crossing was constrained such that $-10 \mathrm{~cm} / \mathrm{s}<\dot{x}<10 \mathrm{~cm} / \mathrm{s}$. Highfidelity orbit propagation incorporating the dynamical models discussed in Section 2.2 was performed using explicit Runge-Kutta-Verner 8(9) and a Dormand-Prince 8(9) integrators in GMAT and STK/Astrogator, respectively. Each stationkeeping $\Delta V$ maneuver was minimized using SQP optimizers including VF13AD from the Harwell library and Matlab's fmincon. To ensure that the spacecraft remained in the vicinity of the Earth-Moon libration point orbits in the near-term, up to four downstream crossings were used during the OCS targeting procedure and stationkeeping maneuvers were executed twice per orbit - approximately once per week.

\subsection{Operational ARTEMIS stationkeeping results}

The OCS was tailored to the ARTEMIS Earth-Moon libration point orbits and was used to successfully maintain the P1 and P2 spacecraft in the vicinity of EM $L_{1}$ and $L_{2}$ during the 11 months of mission operations. The operational stationkeeping maneuvers performed for the ARTEMIS P1 and P2 spacecraft are summarized in Table 2. The actual chronological $\Delta V$ histories for the stationkeeping maneuvers executed for both the ARTEMIS P1 and P2 spacecraft are depicted in Fig. 11. The $\Delta V$ for each maneuver for the P1 spacecraft appears in Fig. 11(a), with the estimated annual maintenance cost for P1 in $L_{2}$ and $L_{1}$ orbit presented in Fig. 11(b) and (c), respectively. Note that, for each maneuver, the estimated annual maintenance cost is extrapolated for one year based on the operational ARTEMIS maneuver schedule and the total stationkeeping $\Delta V$ cost accumulated to date. The stationkeeping $\Delta V$ history for ARTEMIS P2 in the EM $L_{1}$ orbit is depicted in Fig. 11(d). The estimated annual stationkeeping costs for $\mathrm{P} 2$ before and after a change in the coefficient

Table 2

ARTEMIS P1 and P2 stationkeeping results.

\begin{tabular}{lccc}
\hline$\Delta V$ cost & P1 at $L_{2}$ & P1 at $L_{1}$ & P2 at $L_{1}$ \\
\hline Total $\Delta V(\mathrm{~cm} / \mathrm{s})$ & 244.0 & 155.0 & 324.0 \\
Minimum $\Delta V(\mathrm{~cm} / \mathrm{s})$ & 6.96 & 1.17 & 1.33 \\
Maximum $\Delta V(\mathrm{~cm} / \mathrm{s})$ & 22.64 & 27.90 & 37.89 \\
Mean $\Delta V(\mathrm{~cm} / \mathrm{s})$ & 13.51 & 7.21 & 10.85 \\
Standard deviation $(\mathrm{cm} / \mathrm{s})$ & 5.44 & 7.60 & 10.31 \\
\hline
\end{tabular}


a

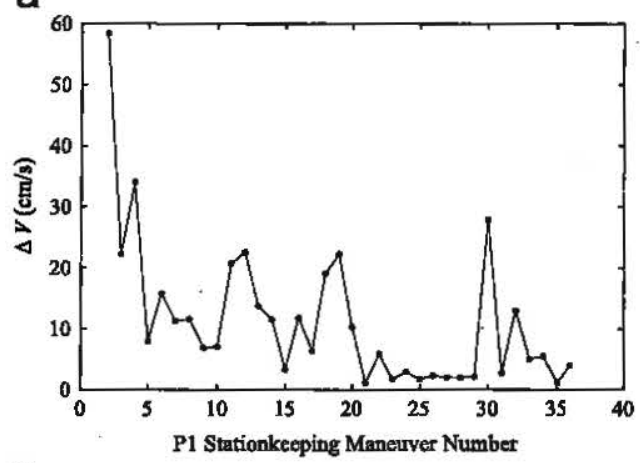

C

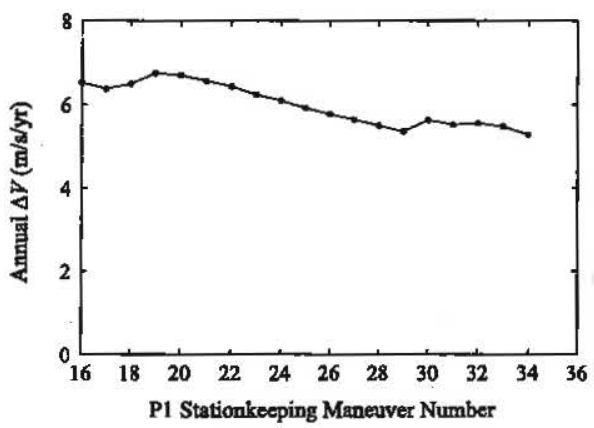

e

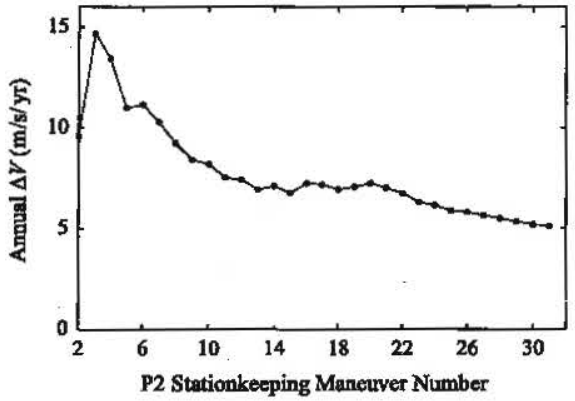

b

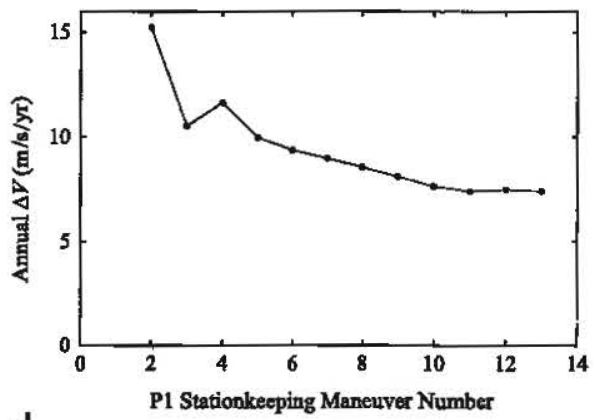

d

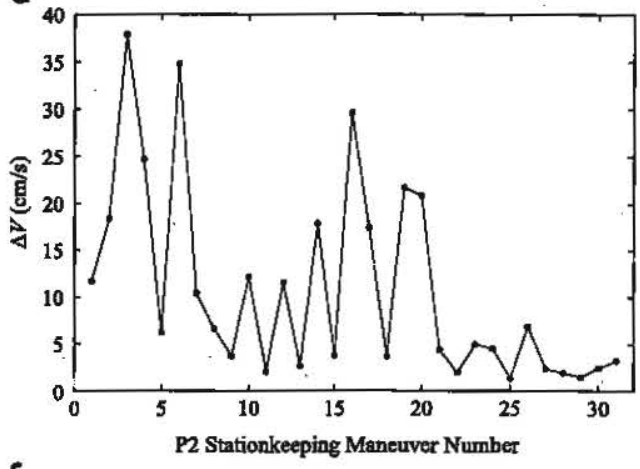

f

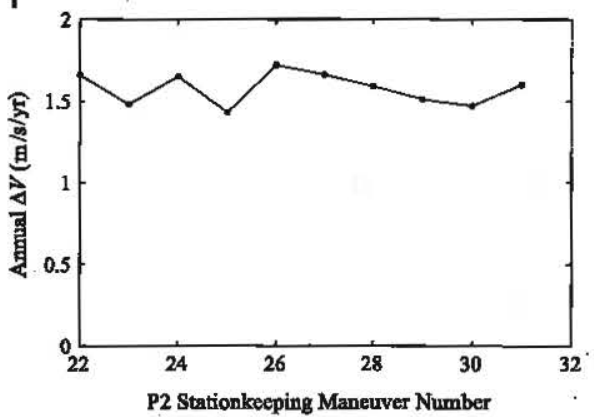

Fig. 11. Operational stationkeeping costs for the ARTEMIS mission. (a) P1 individual SK $\Delta V$, (b) P1 EM $L_{2}$ libration point orbit estimated annual $\Delta V$, (c) P1 EM $L_{1}$ libration point orbit estimated annual $\Delta V$, (d) P2 individual SK $\Delta V$ maneuver, (e) P2 EM $L_{1}$ libration point orbit estimated annual $\Delta V$ (pre- $C_{r}$ change) and (f) P2 EM $L_{1}$ libration point orbit estimated annual $\Delta V$ (post- $C_{r}$ change).

of reflectivity, $C_{r}$, appear in Fig. 11(e) and (f), respectively. Initially, a constant value of $C_{n}$ obtained from analysis conducted before libration point orbit insertion, was utilized for ARTEMIS P2 stationkeeping activities. However, from stationkeeping maneuver \#22 onward, an updated value of $C_{r}$ based on libration point orbit navigation data was implemented; the predictive capability of the dynamical models was improved and, consequently, the stationkeeping costs for the remainder of the ARTEMIS P2 trajectory were significantly reduced. For both the P1 and P2 spacecraft, the general decrease in the estimated annual maintenance cost is attributed to the improved modeling and onboard execution of stationkeeping maneuver thrust arcs.

A summary of stationkeeping costs for ARTEMIS P1 and P2 following libration point orbit insertion, not including the axial correction maneuvers to extend the mission an additional three months [1], are listed in Table 3. Recall that the $\Delta V$ costs per year are extrapolated based on the operational ARTEMIS maneuver schedule
Table 3

ARTEMIS stationkeeping summary.

\begin{tabular}{lll}
\hline$\Delta V$ cost & ARTEMIS P1 & ARTEMIS P2 \\
\hline Total $\Delta V(\mathrm{~m} / \mathrm{s})$ & 3.99 & 3.24 \\
Projected annual $\Delta V, L_{2}(\mathrm{~m} / \mathrm{s})$ & 7.39 & N/A \\
Projected annual $\Delta V, L_{1}(\mathrm{~m} / \mathrm{s})$ & 5.28 & 5.09 \\
\hline
\end{tabular}

and costs observed during the eleven month mission. More detailed ARTEMIS operational stationkeeping $\Delta V$ data, including maneuver epoch information, is provided by Folta et al. [1].

\subsection{ARTEMIS post-mission mode analysis}

From the theoretical perspective, libration point orbit stationkeeping research has been ongoing for over a decade $[23-25,28]$. One particular investigation 
applicable to this analysis is a collaborative effort between Purdue University and Goddard Space Flight Center seeks to further investigate potential links between operational libration point orbit stationkeeping and any orbit stability information available from dynamical systems theory. Analyzing the eigenstructure of a libration point orbit, a process denoted here as mode analysis, supplies information regarding the orbit stability as described in Section 3.2. Note that while the monodromy matrix, $M$, as discussed previously, is defined specifically for periodic orbits, it can be approximated for quasi-periodic trajectories, such as those employed for the ARTEMIS spacecraft, by integrating the orbit for apprcximately one revolution. Application of mode analysis to the ARTEMIS libration point orbits allows for comparison between the theoretical predictions and implemented ARTEMIS stationkeeping maneuver directions computed via the OCS during mission operations.

Using operational ARTEMIS orbit determination solutions along with the operational stationkeeping maneuvers that were actually implemented (designed using the Optimal Continuation Strategy), an approximate monodromy matrix is computed by generating and propagating the state transition matrix, $\Phi\left(t_{2}, t_{1}\right)$, from an initial state for approximately one orbital period. The STM is computed in this analysis by first adding a position or velocity perturbation - that is, $4 \times 10^{-4} \mathrm{~km}$ in position and $1 \times 10^{-4} \mathrm{~cm} / \mathrm{s}$ in velocity - to each component of the initial state and integrating the trajectory forward in time. Then, a finite-difference STM using initial and final state information from numerical a

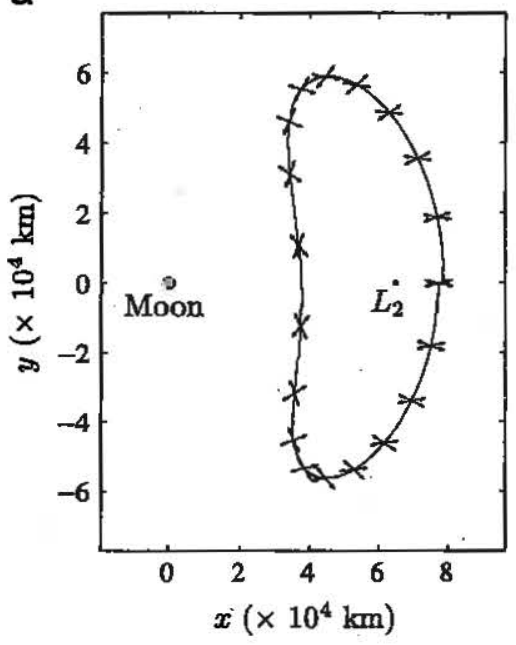

b

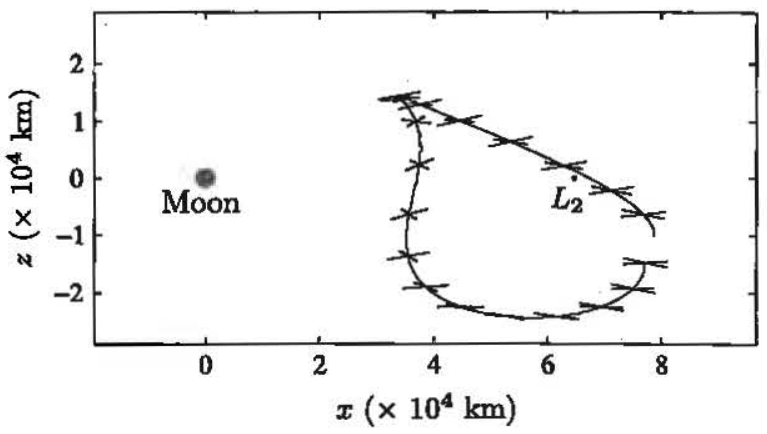

Fig. 12. ARTEMIS P1 EM $L_{2}$ stable and unstable mode directions. (a) $x-y$ projection and (b) $x-z$ projection.

a

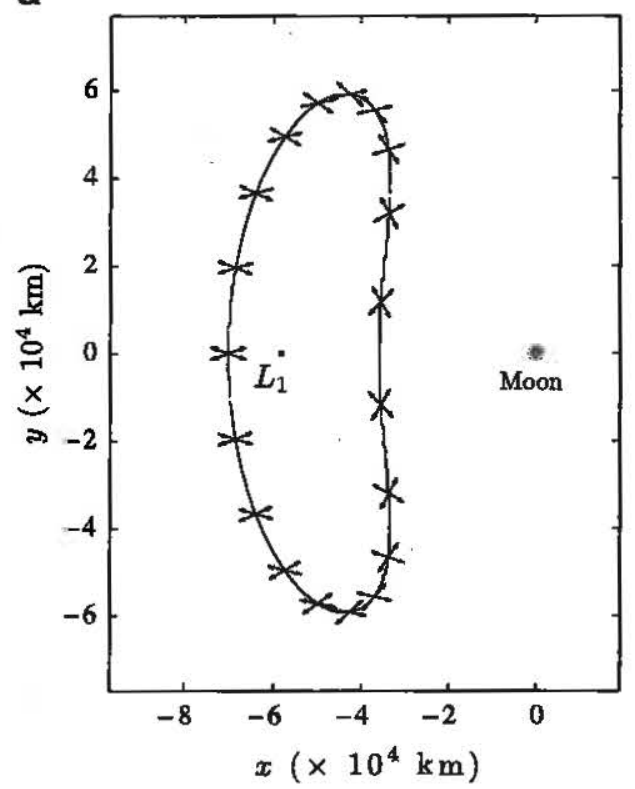

b

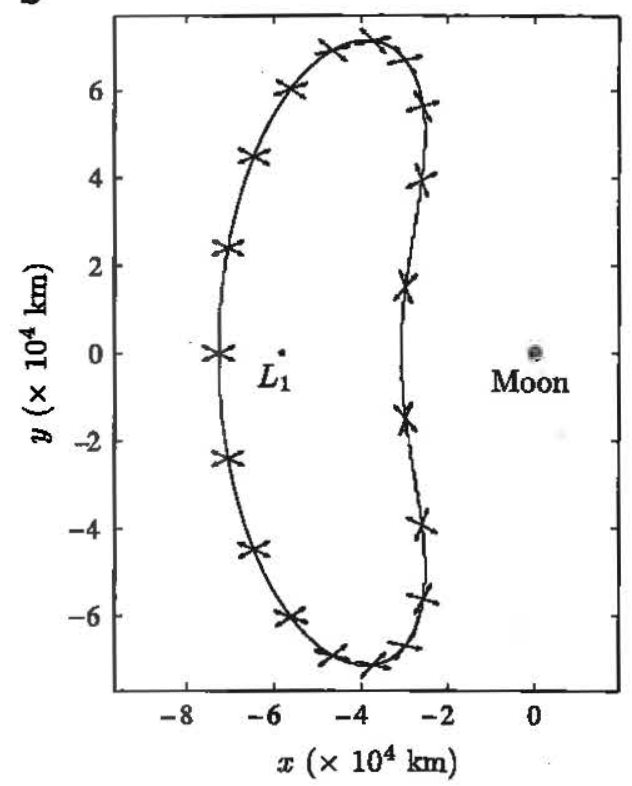

Fig. 13. ARTEMIS P1 and P2 EM $L_{1}$ stable and unstable mode directions. (a) ARTEMIS P1 and (b) ARTEMIS P2. 
integration yields an approximation of the monodromy matrix: The mode information is generated, i.e., the local stable and unstable eigenvalues and eigenvectors are computed, and the actual maneuver direction is compared with the stable/unstable eigenvector information.

As an example, the stable and unstable mode directions associated with the ARTEMIS P1 EM $L_{2}$ orbit over one revolution are represented in Fig. 12 in blue and red, respectively. The stable and unstable eigenvector directions are also displayed for one revolution of the P1 EM $L_{1}$ orbit in Fig. 13(a) and for the P2 EM $L_{1}$ orit in Fig. 13(b). In Figs. 14 and 15 , the stationkeeping $\Delta V$ directions and the stable/unstable mode directions corresponding to the location of a particular maneuver are plotted for the ARTEMIS P1 and P2 spacecraft in EM $L_{1}$ orbit. The angle between the $\Delta V$ vector, as expressed in the EM rotating

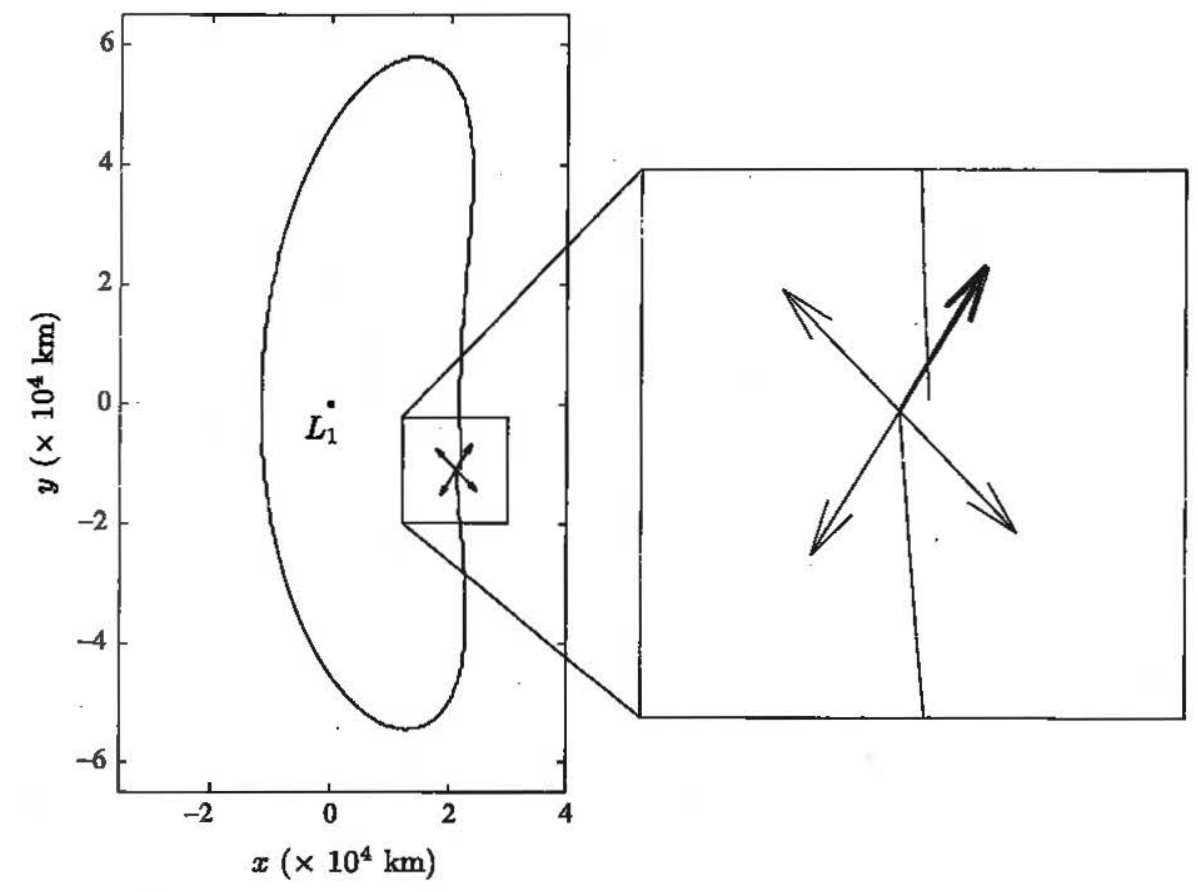

Fig. 14. ARTEMIS P1 SK Maneuver \#21.

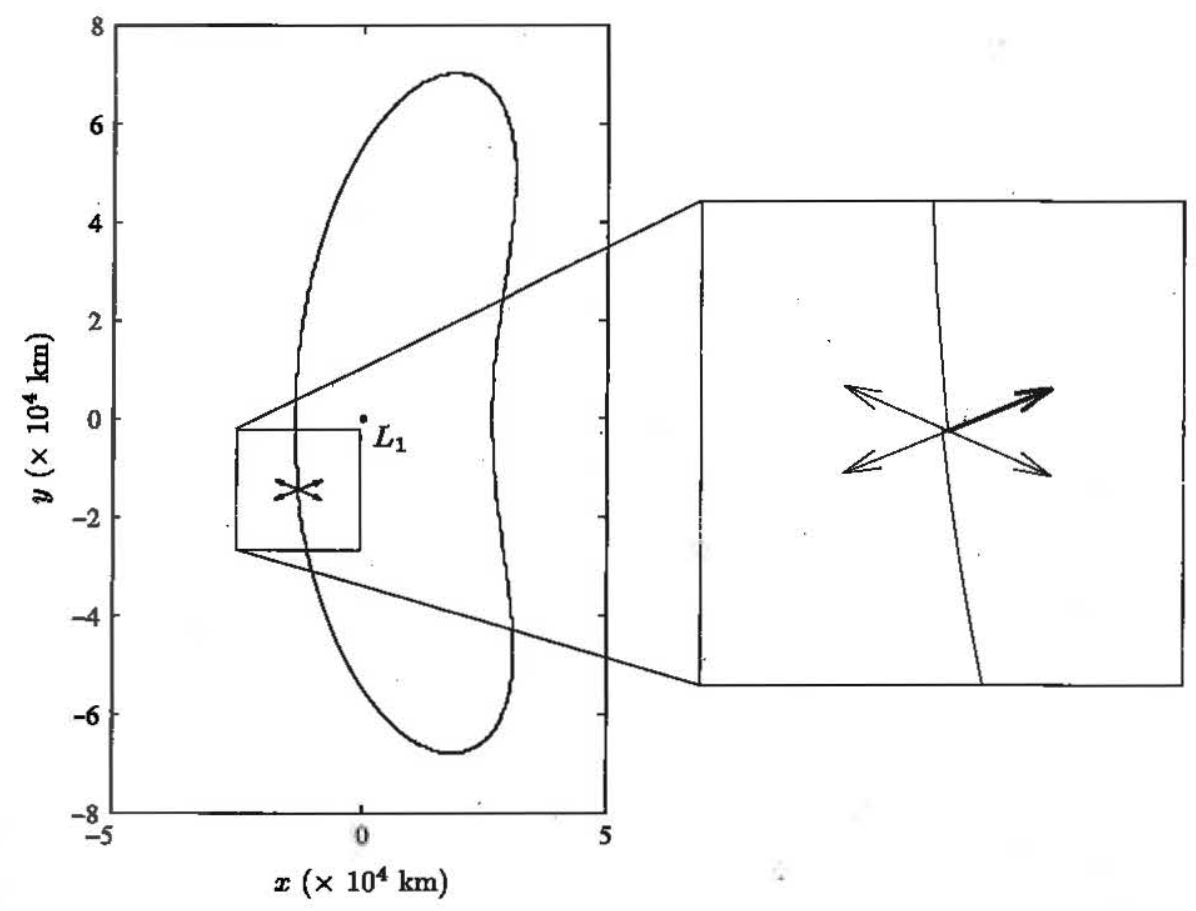

Fig. 15. ARTEMIS P2 SK Maneuver \#4. 

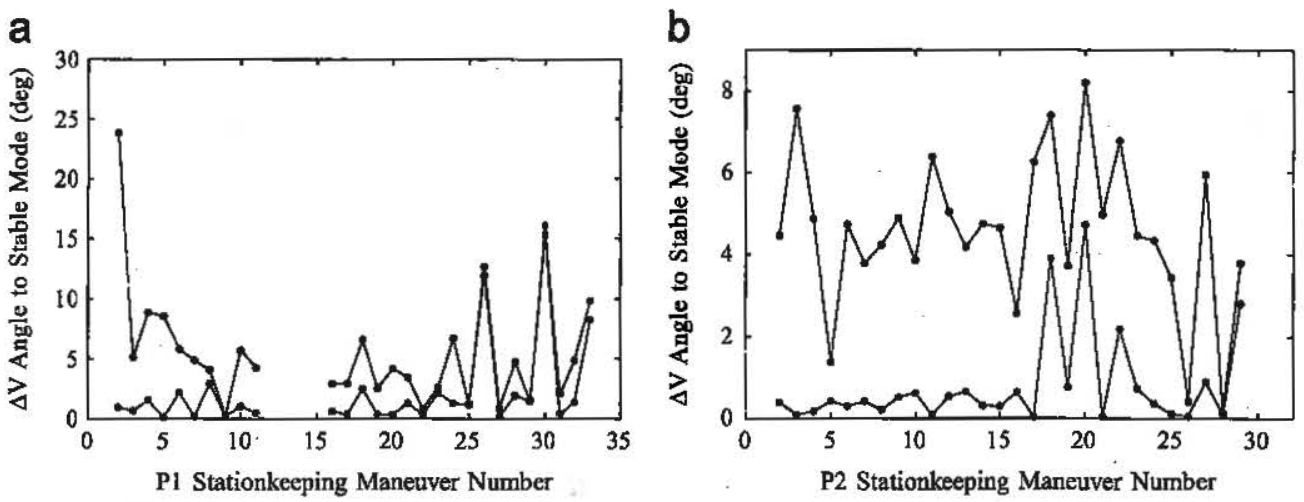

Fig. 16. Total (blue) and in-plane (red) angles between $\Delta V$ vector and the associated stable mode direction. (a) ARTEMIS. P1 and (b) ARTEMIS P2. (For interpretation of the references to color in this figure caption, the reader is referred to the web version of this article.)

coordinate frame, and the stable mode direction is computed from the dot product of the two vectors and is presented for all P1 and P2 stationkeeping maneuvers in Fig. 16. The results of this investigation demonstrate that, even when spacecraft maneuver direction constraints are considered $[1,2]$, the ARTEMIS stationkeeping maneuvers that were executed during mission operations using the Optimal Continuation Strategy are generally aligned with the stable eigenvector direction. This is an interesting result given that previously developed stationkeeping methods incorporating Floquet analysis typically employ stationkeeping maneuvers that align with the unstable mode in such a way that the unstable component of the error is simply canceled. Perhaps not surprisingly, the formulation of the OCS apparently yields maneuvers that strive to return the vehicle to the vicinity of the path dictated by the original libration point orbit insertion condition as the most effective approach. Given the ARTEMIS post-mission mode analysis, it appears likely that stable mode direction information can be incorporated into existing stationkeeping algorithms to compute optimal libration point orbit maintenance maneuvers more quickly and efficiently as demonstrated recently by Pavlak and Howell [29]. At a minimum, the stable mode firection may serve as an initial guess in an optimal strategy. While the full ARTEMIS post-mission mode analysis is still being examined, a basic conclusion is that maneuver placement along the stable mode direction can be used to maintain a libration point orbit by effectively perturbing the trajectory back into the center subspace.

\section{Summary and conclusions}

The ARTEMIS mission utilized high-fidelity modeling and an optimal stationkeeping strategy to successfully maintain the P1 and P2 spacecraft in Earth-Moon $L_{1}$ and $L_{2}$ libration point orbits for eleven months. This research seeks both to summarize the results of ARTEMIS mission operations and to leverage the circular restricted three-body problem and dynamical systems theory in an effort to gain further insight into the evolution of the ARTEMIS libration point orbits and the stationkeeping maneuver design process. The ARTEMIS libration point orbit insertion conditions were dictated by the ballistic Sun-Earth to Earth-Moon transfers that were utilized and, consequently, the ARTEMIS libration point orbits were selected in to enable low-cost transfers between EM $L_{2}$ and $L_{1}$ and, ultimately, into low inclination lunar orbit. Investigating the long-term behavior of the ARTEMIS P1 and P2 orbits using Poincare maps in the circular restricted three-body problem reveals that each of the ARTEMIS EM libration point orbits are, in fact, arcs of large quasi-halo orbits. Analysis of the large quasi-halo orbits available in the CR3B problem demonstrates that these orbits include both high and low $z$-amplitude phases and are well-suited for the ARTEMIS mission because they facilitate both highly out-of-plane and nearly planar arrival and/or departure conditions. With an understanding of the evolution of the trajectories represented on the Poincare map, maps could serve as a useful guide for the selection of orbits with specified characteristics in future mission design applications.

While there are a number of stationkeeping strategies available that incorporate multi-body dynamics, the actual mission applications and constraints must also be considered. The Optimal Continuation Strategy is a general stationkeeping algorithm that is capable of computing low-cost orbit maintenance maneuvers without a reference trajectory and is well-suited for maintaining the ARTEMIS Earth-Moon libration point orbits while satisfying mission constraints. Targeting goals formulated in terms of $x$-velocity at successive $x-z$ plane crossings are utilized to maintain the EM $L_{1}$ and $L_{2}$ for 1-2 revolutions downstream. Incorporating optimization, the required stationkeeping $\Delta V$ can be minimized and has been demonstrated to be only $\sim 5-7 \mathrm{~m} / \mathrm{s}$ per year for the ARTEMIS spacecraft. The optimal stationkeeping maneuver directions yielded by the OCS are further analyzed by completing mode analysis for the ARTEMIS libration point orbits, revealing the directions of the stable and unstable eigenvectors. Optimized maneuver directions computed via the OCS compare closely with the stable mode direction for all stationkeeping maneuvers executed, even when constraints on the feasible spacecraft maneuver directions are considered. With the Earth-Moon libration point orbiting phase of the ARTEMIS mission completed, investigation continues on robust strategies to improve stationkeeping maneuver design for future libration point missions. 


\section{Acknowledgments}

The authors recognize and appreciate the support for efforts at Purdue under NASA contract NNX12AC57G and the NASA Space Technology Research Fellowship (NSTRF) under NASA Grant No. NNX11AM85H. The comments and suggestions from the anonymous reviewers are greatly appreciated and improved the paper significantly.

\section{References}

[1] D. Folta, M. Woodard, D. Cosgrove, Stationkeeping of the first Earth-Moon libration orbiters: the ARTEMIS mission, in: Proceedings of the AAS/AlAA Astrodynamics Specialist Conference, AAS Paper No. 11-515, Girdwood, Alaska, July 2011.

[2] D. Folta, T. Pavlak, K. Howell, M. Woodard, D. Woodfork, Stationkeeping of lissajous trajectories in the Earth-Moon system with applications to ARTEMIS, in: Proceedings of the 20th AAS/AIAA Space Flight Mechanics Meeting, AAS Paper No. 10-113, San Diego, California, February 2010.

[3] T.H. Sweetser, S.B. Broschart, V. Angelopoulos, G.]. Whiffen, D.C. Folta, M.-K. Chung, S.J. Hatch, M.A. Woodard, ARTEMIS Mission Design, Space Sci. Rev. 165 (1-4) (2011) 27-57.

[4] D. Folta, M. Woodard, K. Howell, C. Patterson, W. Schlei, Applications of multi-body dynamical environments: the ARTEMIS transfer trajectory design, Acta Astronautica 73 (2012) 237-249.

[5] D. Sibeck, et al, ARTEMIS science objectives, Space Sci. Rev. 165 (1-4) (2011) 59-91.

[6] T. Pavlak, K. Howell, Evolution of the out-of-plane amplitude for quasi-periodic trajectories in the Earth-Moon system, Acta Astronautica 81 (2) (2012) 456-465.

[7] V. Szebehely, Theory of Orbits: The Restricted Problem of Three Bodies, Academic Press Inc., New York, 1967.

[8] C. Conley, Low energy transit orbits in the restricted three-body problem, Soc. Ind. Appl. Math. J. Appi. Math. 16 (1968) 732-746.

[9] W. Koon, M. Lo, J. Marsden, S. Ross, Heteroclinic connections between periodic orbits and resonance transitions in celestial mechanics, Chaos 10 (June) (2000) 427-469.

[10] G. Gómez, W. Koon, M. Lo, J. Marsden, J. Masdemont, S. Ross, Connecting orbits and invariant manifolds in the spatial restricted three-body problem, Nonlinearity 17 (September) (2004) 1571-1606.

[11] H. Pernicka, K. Howell, Sun-Earth libration point trajectories that avoid the solar exclusion zone, J. Astronaut. Sci. 38 (July-September (3)) (1990) 269-288.

[12] K. Howell, Families of orbits in the vicinity of the collinear libration points, J. Astronaut. Sci. 49 (January-March (1)) (2001) 107-125.

[13] R. Farquhar, A. Kamel, Quasi-periodic orbits about the translunar libration point, Celest. Mech. 7 (1973) 458-473.

[14] D. Richardson, N. Carey, A uniformly valid solution for motion about the interior libration point of the perturbed elliptic-restricted problem, in: Proceedings of the Astrodynamics Specialist Conference, AAS Paper No. 75-021, Nassau, Bahamas, July, 1975.

[15] K. Howell, H. Pernicka, Numerical determination of Lissajous trajectories in the restricted three-body problem, Celest. Mech. 41 (1-4) (1988) 107-124.

[16] E. Kolemen, J. Kasdin, P. Gurfil, Quasi-periodic orbits of the restricted three-body problem made easy, in: New Trends in Astrodynamics and Applications III, AIP Conference Proceedings, vol. 886, Princeton, New Jersey, August 16-18, 2007, pp. 68-77.

[17] D. Grebow, Generating Periodic Orbits in the Circular Restricted Three-body Problem with Applications to Lunar South Pole Coverage, M.S. Thesis, Schoo' of Aeronautics and Astronautics, Purdue University, West Lafayette, Indiana, 2006.

[18] G. Gómez, À. Jorba, J. Masdemont, C. Simó, Dynamics and Mission Design Near Libration Points. Advanced Methods for Collinear Points, vol, III, World Scientific Publishing Co., River Edge, NJ, 2001.

[19] B. Barden, K. Howell, Fundamental motions near collinear libration points and their transitions, ]. Astronaut. Sci. 46 (OctoberDecember (4)) (1998) 361-378.

[20] T. Pavlak, K. Howell, Strategy for long-term libration point orbit stationkeeping in the Earth-Moon system, in: Proceedings of the AAS/AIAA Astrodynamics Specialist Conference, AAS Paper No. 11-516, Girdwood, Alaska, July 2011.

[21] D. Hoffman, Stationkeeping at the Collinear Equilibrium Points of the Earth-Moon System, NASA JSC-26189, September 1993.

[22] R. Farquhar, The Utilization of Halo Orbits in Advanced Lunar Operation, NASA TN D-6365, GSFC, Greenbelt, Maryland, 1971.

[23] C. Renault, D. Scheeres, Statistical analysis of control maneuvers in unstable orbital environments, J. Guid. Control Dyn. 26 (September-October (5)) (2003) 758-769.

[24] K. Howell, T. Keeter, Station-keeping strategies for libration point orbits: target point and Floquet mode approaches, R. Proulx, J. Liu, P. Seidelmann, S. Alfano (Eds.), in: Proceedings of the AAS/AIAA Spaceflight Mechanics Conference 1995, Advances in the Astronautical Sciences, vol. 89, 1995, pp. 1377-1396.

[25] G. Gómez, J. Llibre, R. Martínez, C. Simó, Dynamics and Mission Design Near Libration Points, Fundamentals: The Case of Collinear Libration Points, World Scientific Monograph Series, vol. l, World Scientific Publishing Ltd., Singapore, 2001.

[26] B. Marchand, K. Howell, Formation flight near L1 and L2 in the SunEarth-Moon ephemeris system including solar radiation pressure, in: Proceedings of the AAS/AIAA Astrodynamics Specialist Conference, AAS Paper No. 03-596, Big Sky Montana, August 2003.

[27] D. Folta, F. Vaughn, A survey of Earth-Moon libration orbits: stationkeeping strategies and intra-orbit transfers, in: Proceedings of the AIAA/AAS Astrodymamics Conference, AIAA Paper No. 20044741, Providence, Rhode lsland, August 2004.

[28] K. Howell, H. Pernicka, Station-keeping method for libration point trajectories, J. Guid. Control Dyn. 16 (January-February (1)) (1993) 151-159.

[29] T. Pavlak, K. Howèll, Strategy for optimal, long-term stationkeeping of libration point orbits in the Earth-Moon system, in: Proceedings of the ALAA/AAS Astrodynamics Specialist Conference, AIAA Paper No. 2012-4665, Minneapolis, Minnesota, August 2012 